\title{
SELECCIÓN DE ÁRBOLES DE BOLAINA BLANCA (Guazuma crinita Mart.) COMO CANDIDATOS A ÁRBOLES “PLUS” PARA ENSAYOS DE REJUVENECIMIENTO Y BROTACIÓN
}

\section{SELECTION OF BOLAINA BLANCA (Guazuma crinita Mart.) TREES AS "PLUS" CANDIDATE TREES FOR REJUVENATION AND SPROUTING TRIALS}

\author{
Andrea E. Ramos-Huapaya ${ }^{1}$ y Gilberto Domínguez Torrejón ${ }^{2}$
}

\begin{abstract}
Resumen
La bolaina blanca (Guazuma crinita) es una especie forestal amazónica de importancia económica por su rapidez de crecimiento y diversos usos de su madera. En distintas localidades de la amazonia peruana se está instalando plantaciones, sin un adecuado control de material genético validado. La empresa Consorcio Agroforestal SAC está desarrollando un programa de mejoramiento genético para esta especie, dentro del cual se realizó la selección de árboles candidatos a plus, con base en características fenotípicas y dasométricas para su posterior propagación vegetativa. El objetivo de esta investigación fue caracterizar la respuesta de los árboles a la aplicación de dos tratamientos de rejuvenecimiento (desbaste y descortezado), aplicados a individuos candidatos a árboles plus seleccionados en rodales (menores a 5 años de edad) y plantados en 3 condiciones de sitio ( $\mathrm{km} \mathrm{43,} \mathrm{Navidad} \mathrm{y} \mathrm{Shebonia)} \mathrm{que} \mathrm{se} \mathrm{caracterizan} \mathrm{por}$ tener variaciones ambientales mínimas, ubicados en Puerto Inca (Huánuco). Se realizaron tratamientos de rejuvenecimiento para obtener material de propagación vegetativa; para ello se trataron a 42 árboles (21 inducidos por desbaste y 21, por descortezado); después de 8 semanas (en Navidad y Shebonia) y 10 semanas (en $\mathrm{km} \mathrm{43)} \mathrm{se} \mathrm{contabilizaron} \mathrm{la} \mathrm{totalidad} \mathrm{de} \mathrm{brotes} \mathrm{y} \mathrm{se}$ cosecharon aquellos de aspecto vigoroso. Mediante pruebas no paramétricas se confirmó que los brotes observados en árboles desbastados, tienen una tasa de brotación de 5/1 (5 brotes/árbol tratado). La mayor cantidad de brotes cosechados se obtuvieron de árboles candidatos seleccionados en el sitio Shebonia.
\end{abstract}

Palabras clave: propagación vegetativa, brotación, plantaciones de bolaina, rejuvenecimiento de árboles.

\begin{abstract}
Bolaina blanca (Guazuma crinita) is an Amazonian forest species of economic importance due to its rapid growth and diverse wood uses. In different locations in the Peruvian Amazon plantations are being installed, without adequate control of validated genetic material. Consorcio Agroforestal SAC company is developing a breeding program for this species, in which the selection of plus trees candidates is carried out, based on phenotypic and dasometric characteristics for subsequent vegetative propagation. The objective of this research was to characterize the response of trees to the application of two rejuvenation treatments (roughing and debarking), applied to individual plus candidate trees selected in stands (less than 5 years old) and planted in 3 condition sites ( $\mathrm{km} \mathrm{43,} \mathrm{Navidad} \mathrm{and} \mathrm{Shebonia)} \mathrm{which} \mathrm{are} \mathrm{characterized} \mathrm{by} \mathrm{minimal} \mathrm{environmental}$ variations, located in Puerto Inca - Huanuco). Rejuvenation treatments to obtain vegetative propagation material were performed into 42 trees (21 induced by roughing and 21, by peeling). After 8 weeks (Navidad and Shebonia) and 10 weeks (km 43) all outbreaks were reported and only the vigorous sprouts were harvested. Non parametric tests confirmed that outbreaks in roughing trees have a sprouting rate of 5/1 (5 shoots / treated tree). Most outbreaks were obtained from harvested trees selected candidates at the Shebonia site.
\end{abstract}

Key words: Vegetative propagation, sprouting of buds, plantations of bolaina, rejuvenation of trees. 


\section{Introducción.}

La bolaina blanca (Guazuma crinita Mart.) es una especie forestal amazónica de rápido crecimiento, elevada abundancia natural (reportada en los claros del bosque), de baja tolerancia a la competencia y de alta capacidad de rebrote, (IIAP, 2009). Su madera es usada en carpintería, elaboración de utensilios pequeños y artesanías (Reynel et al., 2003), además en la industria informal de casas pre-fabricadas, de gran demanda por la creciente población.

En sectores de la selva de la región Huánuco (Perú) se está aprovechando en parcelas de regeneración natural y en plantaciones forestales, constituyéndose en una actividad con relevancia económica. Sin embargo, en las plantaciones que se están instalando, se está utilizando, en el mejor de los casos, semillas de procedencia conocida pero no se tiene la certeza de su calidad genética y variabilidad en la descendencia.

Para resolver esta problemática, la empresa Consorcio Agroforestal SAC (CAF SAC) ha iniciado un programa de selección genética de esta especie; con el apoyo financiero del programa Innóvate-Perú (Código del Proyecto: PIPEI-6-P-328-096-13). La finalidad es incrementar la productividad (altura, diámetro y volumen) y mejorar la calidad de los árboles (forma, autopoda y ramificación) en sistemas de plantaciones forestales y agroforestales.

Los programas de mejoramiento genético de especies forestales, se desarrollan a largo plazo y se inician con la selección de árboles sobresalientes (árboles plus). En las condiciones de plantación que se cuenta actualmente, principalmente en la amazonia, en donde el origen de la semilla no ha tenido un control adecuado; la etapa de selección de árboles tiene mucha trascendencia, ya que los árboles seleccionados serán los candidatos a "árbol plus". Esta selección se inicia con la definición de ciertas características fenotípicas, teniendo en cuenta el uso final al que será destinado el producto, en este caso, la madera (Jara citado por Aguirre \& Fassbender, 2013). Vallejos et al. (2010) señalan que la selección se realiza exclusivamente en plantaciones, con base en la expresión fenotípica de caracteres de interés. Por lo tanto, la manifestación de estos caracteres está bajo efectos ambientales y genéticos que podrían ser muy altos en algunos casos y confundir al seleccionador con lo que observe fenotípicamente.

El árbol plus y/o árbol sobresaliente es aquel que posee un fenotipo superior para crecimiento, forma, calidad de la madera u otras características deseables y parece ser adaptable. Sin embargo, no se ha probado su valor genético, aunque las probabilidades de que posea un buen genotipo son por lo general altas (Zobel \& Talbert, citados por Vallejos, 2007). Resende et al., citados por Ramos (2015) afirman que un árbol plus debe mostrar superioridad en volumen y calidad. La superioridad en volumen integra la relación diámetro y altura comercial o yema apical dominante, mientras que la calidad de árbol integra todas las variables cualitativas (diámetro de ramas, ángulo de inserción de ramas, presencia de gambas o aletones, grano en espiral, sanidad, entre otras).

Posterior a la selección de árboles de apariencia sobresaliente, se procede a la aplicación de tratamientos de rejuvenecimiento (como: desbaste o corte total del árbol y anillado o descortezado) con la finalidad de obtener material juvenil para la propagación vegetativa, como por ejemplo estacas y posteriormente mediante multiplicaciones clonales sucesivas, realizar ensayos de progenie para validar la superioridad de los árboles sobresalientes seleccionados y la trasmisión genética de las características deseables por las cuales se calificó. Mesén et al., (1996) sostienen que la propagación vegetativa ofrece los medios para lograr las mayores ganancias genéticas en menos tiempo.

Xavier et al., (2013) definen al rejuvenecimiento como una forma de revertir en la planta el estado adulto a su estado juvenil, recuperando la capacidad de totipotencia; además la describen como prácticas culturales que tienen como objetivo propiciar mayor vigorosidad fisiológica en la brotación de plantas, de forma que proporcione material vegetativo vigoroso y sensible a la propagación vegetativa. El rejuvenecimiento (de partes adultas de los árboles) se obtiene al realizar el corte total o parcial de los árboles, pues los retoños exhiben características juveniles, y alta capacidad de enraizar (Gutiérrez, 1995). El tratamiento del desbaste es de carácter destructivo, consiste en cortar los árboles, dejando un tocón a $15 \mathrm{~cm}$ de altura, del cual se obtendrá material vegetativo, (Gutiérrez et al., 1994). Assis \& Rodríguez (2014) sostienen que el desbaste es el tratamiento más simple de rejuvenecimiento, sin embargo, no todas las especies generan brotes después del corte. Sostienen además, que los brotes que surgen de la región basal tienden a presentar características morfológicas y fisiológicas más juveniles (incluyendo el enraizamiento).

El tratamiento del descortezado, consiste en estimular la regeneración de brotes en el árbol en pie, mediante la realización de cortes en la corteza de la base. Alfenas et al., (2004) sostienen que este tratamiento es de fácil ejecución, en la mayoría de casos su efecto ha dado buenos resultados (especialmente con especies de eucalipto). El fundamento es que el corte interrumpe el flujo basípeto de auxinas y otras sustancias, y en ocasiones estimula la brotación de yemas juveniles dormantes que se encuentran por debajo del corte (Mesén, 1998).

La aplicación y desarrollo de programas de mejoramiento genético, pretende lograr altos rendimientos en producción de madera de calidad por hectárea, en menor tiempo y al menor costo posible. Sin embargo, es un proceso a largo plazo y requiere de 
investigación constante. La presente investigación permitirá sentar las bases para iniciar el programa de mejoramiento genético de Guazuma crinita, el cual se iniciará con la selección de árboles para la propagación vegetativa o asexual.

La selección de árboles candidatos a plus de una especie forestal, no es una práctica común en el Perú, se requiere establecer criterios de calificación de los parámetros deseables para cada especie y el producto que se quiera aprovechar de ella; se desconoce las técnicas de propagación asexual y la calidad del material que se debe inducir para disponer de semillas vegetativas aplicando técnicas de rejuvenecimiento. Es por ello que se realizó la presente investigación, con el objetivo de caracterizar la respuesta a la aplicación de tratamientos de rejuvenecimiento de árboles seleccionados como individuos candidatos a “árbol plus” localizados en 3 condiciones de sitio.

\section{Materiales y métodos.}

Localización.

El estudio se realizó en el distrito de Puerto Inca ( $9^{\circ} 40^{\prime} 31^{\prime \prime}$ S, 75²7’01”O), a la altitud promedio de 200 msnm, provincia de Puerto Inca, región Huánuco, Perú. Al ámbito de estudio le corresponde la zona de vida de bosque muy húmedo pre montano (bmh - P), presentando una temperatura media de $23{ }^{\circ} \mathrm{C}$, y una precipitación anual de $2500 \mathrm{~mm}$ con 3 meses de época seca y una humedad relativa de $80 \%$. Los suelos son franco arenoso, profundo y drenado, (ONERN, 1976). Los árboles candidatos a plus fueron

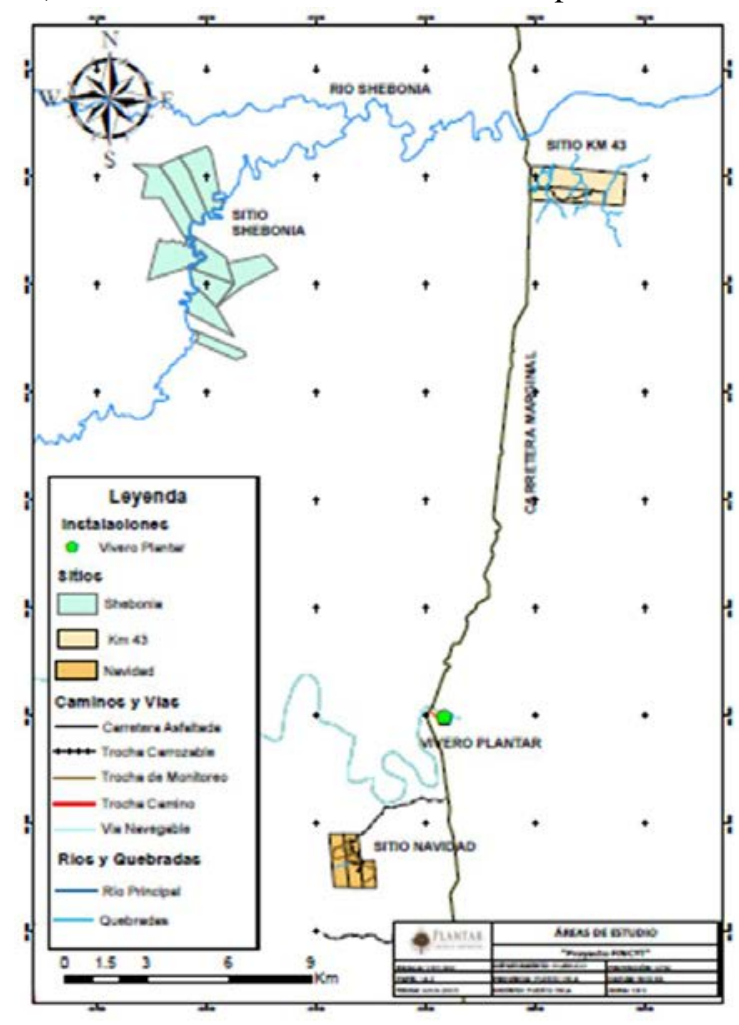

Figura 1. Ubicación de las 3 condiciones de sitio.

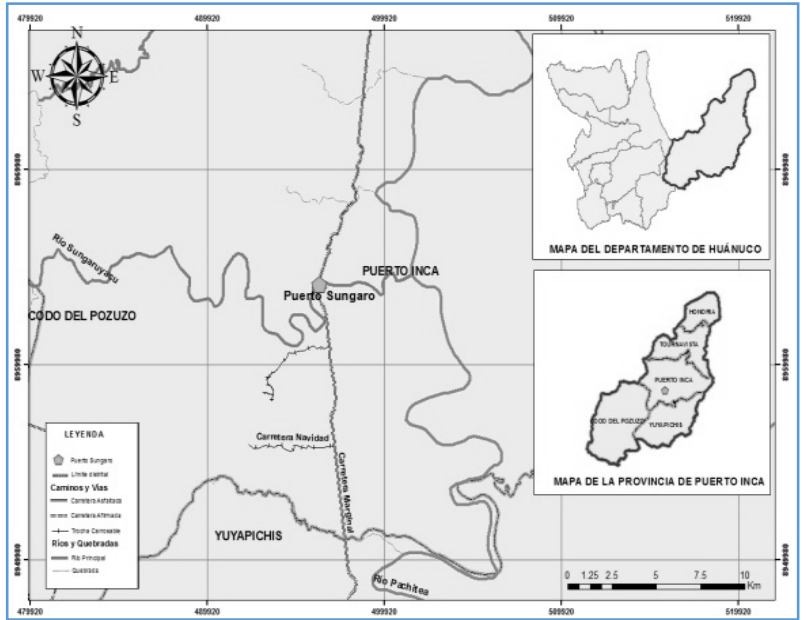

Figura 2. Ubicación de Puerto Súngaro.

seleccionados en plantaciones de bolaina blanca (menores a 5 años de edad) instaladas y manejadas por la empresa Consorcio Agroforestal SAC, en los lugares o sitios conocidos como: "km 43", "Navidad" y "Shebonia".

Las plantaciones (Figura 1) fueron instaladas con plantas de calidad (tallo recto, sanas, más de $25 \mathrm{~cm}$ de longitud, estado nutricional óptimo) producidas en el Vivero Forestal de alta tecnología de la empresa CAF SAC, ubicado en Puerto Súngaro (Figura 2). Estas plantas provienen de semillas cosechadas en rodales semilleros localizados en Pucallpa (proyecto PROSEMA ICRAF).

Caracterización de los sitios.

En la Tabla 1 se presentan las características de los sitios trabajados.

La superficie total de las plantaciones es de 40.6 ha y la densidad promedio es de 1111 árboles por hectárea (distanciamiento $3 \times 3 \mathrm{~m}$ ). Respecto a los antecedentes de las plantaciones, se tiene el bosque secundario joven que fue identificado como el terreno abandonado y en proceso de repoblamiento de vegetación silvestre (arbórea efímera y arbustiva agresiva). Mientras que el bosque secundario maduro

Tabla 1. Caracterización de los sitios.

\begin{tabular}{|c|c|c|c|}
\hline Sitio & km 43 & Navidad & Shebonia \\
\hline Superficie (ha) & 21.9 & 8.6 & 10.1 \\
\hline Edad (meses) & 44 & 44 & 56 \\
\hline Edad (años) & 3.7 & 3.7 & 4.7 \\
\hline Fisiografía & $\begin{array}{l}\text { Planicie } \\
\text { coluvial, } \\
\text { piedemonte }\end{array}$ & $\begin{array}{l}\text { Planicie } \\
\text { coluvio- } \\
\text { aluvial }\end{array}$ & $\begin{array}{l}\text { Planicie } \\
\text { aluvial, } \\
\text { terraza media }\end{array}$ \\
\hline $\begin{array}{l}\text { Profundidad de } \\
\text { hojarasca }(\mathrm{cm}) \\
\text { Antecedentes }\end{array}$ & $3-5$ & $3-5$ & $3-5$ \\
\hline $\begin{array}{l}\text { Tipo de bosque } \\
\text { secundario }\end{array}$ & $\begin{array}{l}\text { Joven } \\
\text { maduro } \\
(65 \%)\end{array}$ & Joven & Maduro \\
\hline Uso anterior & $\begin{array}{l}\text { pastura } \\
\text { para } \\
\text { ganado }\end{array}$ & $\begin{array}{l}\text { pastura } \\
\text { para } \\
\text { ganado }\end{array}$ & $\begin{array}{l}\text { pastura } \\
\text { ganado }\end{array}$ \\
\hline
\end{tabular}


Tabla 2. Mantenimiento de los sitios.

\begin{tabular}{|c|c|c|c|}
\hline \multirow[b]{2}{*}{ Sitio } & \multicolumn{3}{|c|}{ Mantenimientos/ Edad de la plantación } \\
\hline & Año 1 & Año 2 & Año 3 \\
\hline $\mathrm{Km} 43$ & mantenimientos: & mantenimientos: & 3 mantenimientos: \\
\hline \multirow{2}{*}{ Navidad } & control de malezas & control de malezas & control de malezas \\
\hline & (desmalezado $\quad+$ & (desmalezado + & (desmalezado + \\
\hline Shebonia & control químico) & control químico) & control químico) \\
\hline
\end{tabular}

fue identificado como el terreno que presentó vegetación arbórea ya establecida de lento crecimiento.

Según los registros históricos, las plantaciones no han recibido ningún tipo de fertilización, solo se han ejecutado actividades de mantenimiento como control de malezas (desmalezado y control químico). Las actividades de mantenimiento se intensificaron en el $1^{\circ}$ año de la plantación, para luego disminuir su frecuencia (Tabla 2). Es preciso mencionar que el control químico incluye la aplicación de herbicidas de acción directa sobre la vegetación herbácea, sin alterar significativamente las características del suelo.

El análisis de caracterización de suelo reportan diferencias (no significativas) entre los sitios, sin embargo algunas diferencias podrían deberse al uso anterior del sitio (bosque secundario joven y maduro) (Tabla 3).

Los sitios seleccionados se caracterizan por ser sitios donde los efectos de las fuentes genéticas de variación a nivel macro (clima, suelos, edad) son mínimos y los efectos por la intervención antrópica son similares (control de malezas). Mesén (1998) señala que la selección de árboles para programas de mejoramiento genético, es más eficiente en plantaciones puras que crecen en sitios homogéneos, donde las variaciones ambientales son mínimas, puesto que lo deseable es que la variación fenotípica refleje más fielmente la variación genética.

Selección de árboles.

En la selección de los árboles candidatos se consideraron criterios establecidos previamente, los cuales se definieron con base en su heredabilidad (carácter fenotípico) y su fácil medición (carácter dasométrico). Los caracteres más representativos fueron: la manifestación genética (DAP) y el estado fitosanitario (el cual se expresa en el periodo juvenil y repercute en el periodo adulto). Los caracteres que se aplicaron en el mejoramiento fueron: diámetro a la altura del pecho (DAP), altura total $\left(\mathrm{H}_{\mathrm{t}}\right)$, estado fitosanitario $\mathrm{y}$ calidad del fuste. Se seleccionaron 42 árboles candidatos a plus distribuidos en los 3 sitios (15 árboles: sitio $\mathrm{km} \mathrm{43;} 14$ árboles: sitio Navidad y 13 árboles: sitio Shebonia).

En la selección de los árboles candidatos a plus se utilizó: GPS Garmin MAP 78SC Martine, motosierra Husqvarma 236 E, machetes, serrucho cola de zorro y un formón de 1".

La población de árboles candidatos seleccionados del sitio Shebonia reportaron los valores más altos para los criterios de selección: diámetro a la altura del pecho (DAP) y altura total (Ht) (Tabla 4); se observa una clara diferencia sustentada en la edad de plantación de Shebonia frente a las otras dos, debido a que cuenta con 1 año más desde su instalación.

Tabla 4. Caracterización de la población seleccionada.

\begin{tabular}{lllllll}
\hline & \multicolumn{3}{c}{ DAP $(\mathrm{cm})$} & \multicolumn{3}{c}{ Ht $(\mathrm{m})$} \\
\cline { 2 - 7 } Sitio & Media & Mín. & Máx. & Media & Mín. & Máx. \\
\hline Km 43 & 21.1 & 19.4 & 24.8 & 17.6 & 16.3 & 19.0 \\
Navidad & 19.5 & 16.6 & 21.6 & 17.3 & 14.3 & 20.8 \\
Shebonia & 23.4 & 20.7 & 27.1 & 20.5 & 17.8 & 25.1 \\
\hline
\end{tabular}

\section{Tratamientos.}

Se aplicaron 2 tratamientos de rejuvenecimiento: desbaste (corte total del árbol) y descortezado (anillado) al inicio de la época lluviosa (noviembre y diciembre, 2014), lo que permitió la acumulación de humedad en el suelo. Cada tratamiento fue aplicado en el $50 \%$ de la población seleccionada. Previo a su ejecución, se agregó (de manera aleatoria, pero manteniendo el registro de la actividad) $70 \mathrm{~g}$ de fertilizante nutricional (NPK) a 31 árboles candidatos seleccionados. El fertilizante estuvo conformado por la mezcla de: Cloruro de potasio (K), Fosfato di amónico (P) y Urea (N), en la proporción de 20-20-20 y fue aplicada a una profundidad de $30 \mathrm{~cm}$, para facilitar el contacto con las raíces laterales absorbentes.

El desbaste consistió en la tala del árbol candidato seleccionado, se dejó un tocón de $35 \mathrm{~cm}$ de altura, el corte fue biselado para que la humedad acumulada no afecte de manera negativa al tocón, ya que se iniciaba la época lluviosa. El descortezado consistió en realizar

Tabla 3. Análisis de caracterización de suelo.

\begin{tabular}{|c|c|c|c|c|c|c|}
\hline Sitio & $\begin{array}{c}\text { pH(1:1) } \\
\text { Reacción }\end{array}$ & $\begin{array}{c}\text { CE (es) } \\
\text { Salinidad } \\
\end{array}$ & $\begin{array}{c}\text { M.O. (\%) } \\
\text { Clasificación }\end{array}$ & $\begin{array}{c}\text { P (ppm) } \\
\text { Disponible }\end{array}$ & $\begin{array}{c}\text { K (ppm) } \\
\text { Disponible }\end{array}$ & Clase Textural \\
\hline $\mathrm{km} 43$ & $\begin{array}{l}\text { ligeramente alcalino- } \\
\text { ligeramente ácido }\end{array}$ & $\begin{array}{l}\text { muy ligeramente } \\
\text { salino }\end{array}$ & bajo & bajo & medio & franco arcilloso - arcilloso \\
\hline Navidad & $\begin{array}{l}\text { moderadamente } \\
\text { ácido-neutro }\end{array}$ & $\begin{array}{l}\text { muy ligeramente } \\
\text { ácido }\end{array}$ & medio & bajo & medio & franco - arcilloso \\
\hline Shebonia & $\begin{array}{l}\text { ligeramente ácido- } \\
\text { fuertemente ácido }\end{array}$ & $\begin{array}{l}\text { muy ligeramente } \\
\text { salino }\end{array}$ & bajo & bajo & Bajo & franco arcilloso - arcilloso \\
\hline
\end{tabular}


cortes (en forma de $\mathrm{V}$ invertida) en la corteza de la base del árbol a $35 \mathrm{~cm}$ sobre el nivel del suelo, en dirección este-oeste. La longitud promedio de los cortes fue equivalente al 50\% del diámetro del árbol, el ancho fue de $5 \mathrm{~cm}$ y el espesor fue de $1.5-2 \mathrm{~cm}$, según recomendación de Saldaña citado por Ramos (2015)

Se realizó el monitoreo de la brotación a los árboles tratados, iniciándose con la limpieza del área (en un radio de $7 \mathrm{~m}$ ) y la aplicación de un insecticida (contra el ataque de hormigas). El monitoreo se realizó cada 15 días. Después de 8 semanas (sitio Navidad y Shebonia) y 10 semanas (sitio $\mathrm{km} \mathrm{43)} \mathrm{se} \mathrm{realizó} \mathrm{el}$ conteo de brotes (brotes observados) y la cosecha de los brotes de mejor aspecto (de características deseables: brotes cosechados), para de ellos obtener estacas y someterlas a tratamientos de enraizamiento.

En el monitoreo de la brotación y cosecha de brotes se utilizó: Vernier Tactix, tijera de podar, cooler térmico, hielo seco, regla metálica de $60 \mathrm{~cm}$, papel periódico y mapas de los sitios.

Los datos fueron analizados mediante pruebas no paramétricas (distribuciones Chi-cuadrado): prueba de distribución multinomial y prueba de independencia, al nivel de confiabilidad de 95\%. Para el análisis de los datos se utilizó el programa estadístico Minitab 14 y el software Microsoft Excel (versión 2010).

\section{Resultados.}

Tratamientos de rejuvenecimiento de los árboles candidatos.

La distribución de los tratamientos se observa en la metodología, (Figura 3).

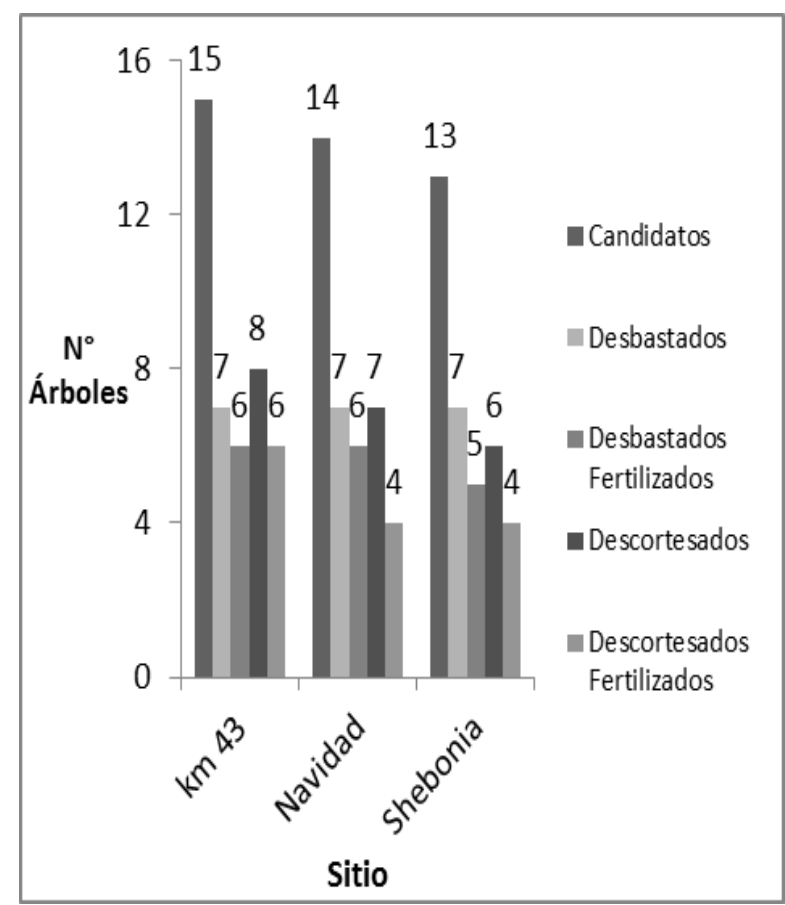

Figura 3. Aplicación de tratamientos y adición de fertilizante.
A través del monitoreo, se reportó la presencia de hongos en la base de algunos tocones, y su origen fue la humedad acumulada en la madera y en el suelo. En algunos árboles descortezados se reportó la cicatrización de los cortes realizados, es decir los cortes no fueron profundos ya que no se interrumpió el flujo basípeto y/o el árbol mostró un excelente comportamiento ante las lesiones.

Caracterización de los brotes.

La emisión de los brotes se inició a los 30 días después de la ejecución de los tratamientos de rejuvenecimiento (esto se reportó para los sitios km 43 y Shebonia). Se observó además que de una yema se formaron como mínimo 1 brote; el promedio de brotes observados por árbol fue 1.1 para el sitio km 43; para el sitio Navidad fue de 2.6 y para el sitio Shebonia fue 3.3, (Tabla 5). Sin embargo, en los árboles con múltiples brotes por yema, solamente de 2 a 3 presentaron un rápido crecimiento, lo cual afectó a las demás; es decir existió una competencia por luz entre los mismos.

Tabla 5. $\mathrm{N}^{\circ}$ de brotes/árbol.

\begin{tabular}{lccc}
\hline & \multicolumn{3}{c}{ Brotes/árbol } \\
\cline { 2 - 4 } Sitio & Promedio & $\mathrm{N}^{\circ}$ Mínimo & $\mathrm{N}^{\circ}$ Máximo \\
\hline km 43 & 1.1 & 1 & 3 \\
Navidad & 2.6 & 1 & 7 \\
Shebonia & 3.3 & 1 & 11 \\
\hline
\end{tabular}

Se realizó la cosecha de los brotes de características deseables (buena pigmentación, vigor, sanidad y carencia de ramificación axilar) presentes en los árboles candidatos. Sin embargo, no todos los árboles reportaron brotes a pesar de la adición de fertilizantes. Los brotes cosechados y de características deseables presentaron una longitud de 10 - $35 \mathrm{~cm}$ y un diámetro comprendido entre 2 - 8 $\mathrm{mm}$. Del total de árboles candidatos a los cuales se les aplicó las técnicas de rejuvenecimientos, solo 21 árboles presentaron brotes juveniles de características deseables para ser cosechados.

Los árboles tratados por desbaste presentaron mayor cantidad de brotes (observados y cosechados) que los árboles tratados por descortezado (Tabla 6), esto confirma lo señalado por Mesén (1998) sobre la efectividad del tratamiento de desbaste frente al descortezado.

Los árboles candidatos seleccionados del sitio Shebonia reportan la mayor cantidad de brotes observados y cosechados; esto sugiere que las condiciones de este sitio son favorables para su mejor desarrollo, además puede afirmarse que estos árboles candidatos tienen una alta capacidad de brotación, (Figura 4).

Se efectuó la prueba de ajuste a la distribución multinomial para cada tratamiento $(\alpha=0,05)$, siendo la hipótesis a comprobar: la cantidad de brotes en árboles tratados por desbaste se encuentran en la 
Tabla 6. Brotes observados y cosechados.

\begin{tabular}{lcc}
\hline \multicolumn{2}{c}{ Tratamiento de } & \multicolumn{2}{c}{ BROTES } \\
Rejuvenecimiento & Observados & Cosechados \\
\hline Desbaste & 107 & 88 \\
Descortezado & 9 & 7 \\
TOTAL & $\mathbf{1 1 6}$ & $\mathbf{9 5}$ \\
\hline
\end{tabular}

proporción de 5 y la cantidad de brotes en árboles tratados por desbaste se encuentran en la proporción de 2 .

Respecto a la adición de fertilizante, se efectuó la prueba de independencia para cada tratamiento $(\alpha=$ $0,05)$, siendo la hipótesis a comprobar: la adición de fertilizante y la cantidad de brotes por cada sitio son independientes, es decir no están relacionadas entre sí.

\section{Discusión.}

Árbol candidato.

La selección se realizó en función a las características fenotípicas y dasométricas observadas, cuantificadas y calificadas. Según Bello (1997), esta definición (forma de selección) le corresponde a un árbol candidato, es decir, un árbol candidato es aquel individuo que por sus características fenotípicas deseables ha sido seleccionado para evaluarlo, pero que aún no se ha valorado ni sometido a pruebas genéticas; por lo tanto, todavía no es considerado como árbol plus.

La superioridad fenotípica que reportaron los

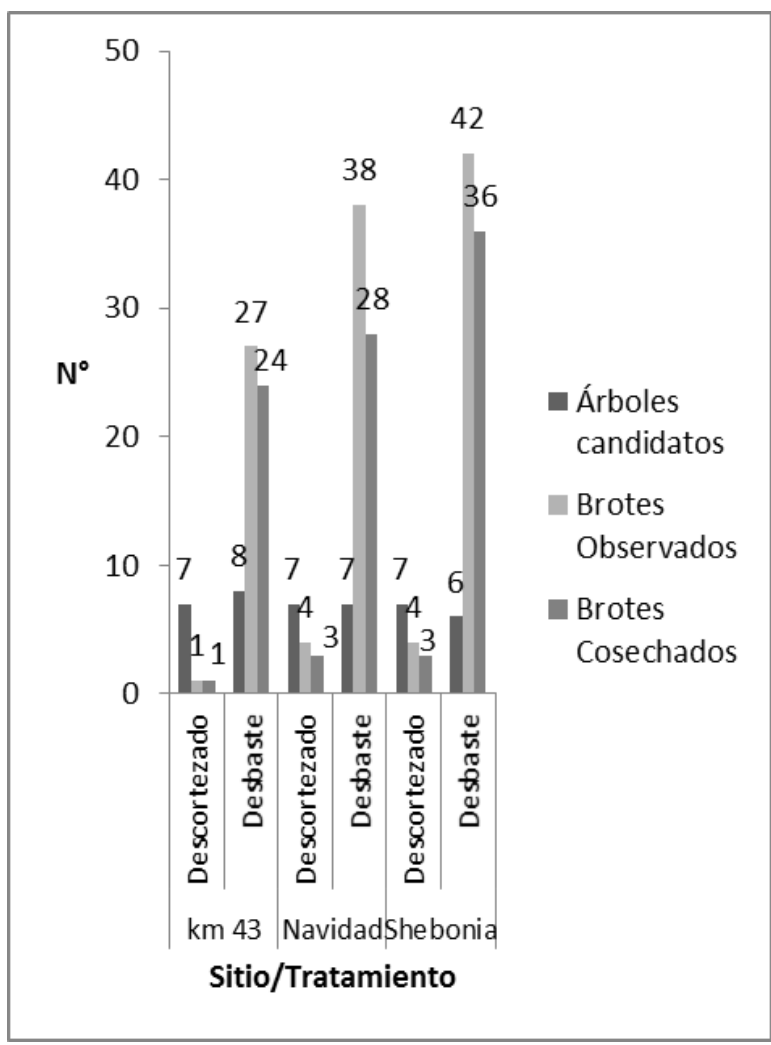

Figura 4. Brotes observados y cosechados por sitio. árboles seleccionados no fue comparada con los árboles vecinos, y tampoco es posible estimar (con la información registrada) la ganancia genética esperada, ni su superioridad en volumen y en calidad, información que define a un árbol plus, según Vallejos et al., (2010) y Resende et al., citado por Ramos (2015). Por lo tanto, según la metodología de selección aplicada, los árboles seleccionados para el programa solo son candidatos a árbol plus hasta validar sus características mediante ensayos de progenie y/o ensayos clonales.

Por lo tanto, puede definirse a un árbol candidato como aquel individuo de apariencia sobresaliente (como sanidad y calidad de fuste), de características dasométricas no cuantificadas pero calificadas como altas y/o por encima del promedio poblacional, es decir es aquel que muestra superioridad fenotípica comparada con sus vecinos más cercanos.

Tratamiento de rejuvenecimiento de árboles candidatos.

Respecto a la época del año, en la cual se debe realizar los tratamientos de rejuvenecimiento, Gutiérrez et al., (1994), por su experiencia con eucalipto, recomiendan la realización del desbaste a finales de la época lluviosa o a comienzos de primavera; Torres (1987) recomienda evitar la acumulación de agua sobre el tocón, ya que este será un medio de cultivo de hongos que atacan la madera y generan la pudrición de la misma. Según la recopilación de Villegas (2003) sobre brotes de eucalipto, la falta de humedad en el suelo puede producir la mortandad de los tocones o pérdidas de vigor en los brotes. Por su parte Mesén (1998) menciona que la humedad estimula la producción de brotes vigorosos. Nogueira et al., (2011) concuerdan con los autores mencionados, y sostienen que los árboles tratados por desbaste durante el periodo lluvioso tienen mayor oportunidad a presentar brotes, que los árboles tratados en periodos secos. Respecto al tratamiento del descortezado, Alfenas et al., (2004) señalan que se obtiene una menor respuesta a la brotación en épocas anteriores al inicio de lluvias, puesto que las plantas presentan mayor actividad metabólica. Recomiendan además considerar la velocidad y la eficiencia de cicatrización del corte, la cual varía entre los árboles. Por lo tanto, queda claro que la humedad influye en la aparición de los brotes, sin embargo el exceso puede causar la muerte de los árboles tratados.

Sobre el monitoreo de la brotación, Nogueira et al., (2011) recomiendan considerar actividades de control para evitar el ataque de hormigas cortadoras y otros insectos sobre los brotes, lo que conllevaría a la pérdida de los mismos.

Los resultados muestran que no todos los árboles tratados generaron brotes; además en muchos casos, las características de los brotes no fueron las deseables para continuar con el desarrollo del programa. 
DRFFMA (1991) señala que los tocones de Guazuma crinita generados por el desbaste, reportan mayor capacidad de generar brotes cuando el corte se encuentra sobre los $20-25 \mathrm{~cm}$ del nivel del suelo. Trujillo (2009) señala que cuando el corte se realiza a ras del suelo, el porcentaje de brotación aumenta y si es muy alto, los brotes caen fácilmente, por ello recomienda que la altura de corte sea la más baja posible $(20-30 \mathrm{~cm})$. Sin embargo, Assis \& Rodríguez (2014) según su experiencia con eucalipto, recomiendan que en el tratamiento del desbaste, se retire una capa de suelo de la base del árbol y que el corte se realice entre los 5-10 cm por encima de la profundidad de inserción de las raíces, esto con la finalidad de obtener brotes con mayor juvenilidad.

En el tratamiento del desbaste, el corte se ejecutó a $35 \mathrm{~cm}$ sobre el nivel del suelo, para evitar la acumulación de agua y consecuentemente la pudrición del tocón y/o la aparición de organismos patógenos que pudieran limitar la formación de brotes. Se tuvo esta consideración puesto que se acercaba la época lluviosa.

Caracterización de los brotes.

Según la experiencia de Saldaña citado por Ramos (2015), la cantidad de brotes generados en árboles de Guazuma crinita tratados por desbaste, es mayor a la generada por descortezado, la cual se expresa en la relación de 5 a 2, respectivamente. En el desarrollo el programa fue posible comprobar la efectividad del desbaste, y mediante el análisis estadístico, es posible afirmar que en los 3 sitios ( $\mathrm{km} \mathrm{43,} \mathrm{Navidad} \mathrm{y}$ Shebonia) los brotes observados en árboles tratados por desbaste, se encuentran en la proporción de 5 (5 brotes por árbol tratado).Sin embargo, no se puede afirmar que los brotes observados en árboles tratados por descortezado se encuentran en la proporción de 2 .

Respecto al efecto de la adición de fertilizante, es posible afirmar que la adición de fertilizantes (NPK) influyó en la cantidad de brotes observados en cada sitio ( $k m$ 43, Navidad y Shebonia). Sin embargo, debe considerarse la presencia de estos elementos en el suelo (Tabla 3), que a pesar de encontrarse en cantidades bajas a medias (Fósforo y Potasio), pudieron fomentar la aparición de los brotes, puesto que participan en el metabolismo de las células vegetales, (Zabaleta, 1992). Saldaña citado por Ramos (2015) señala que existe una relación positiva entre la aplicación de fertilizantes y los brotes generados, esto lo observó en brotes de Guazuma crinita. Por su parte, Yepes et al., (2010) obtuvieron diferencias significativas entre la aplicación de fertilizante (urea) a árboles de Swietenia macrophylla y la emisión de brotes (cantidad y longitud). Los resultados obtenidos confirmarían la relación directa entre el fertilizante aplicado y los brotes observados, sin embargo no todos los brotes observados reportaron las características deseables para ser cosechados.
No todos los brotes observados fueron cosechados, debido a la variabilidad reportada en las características requeridas para obtener estacas y continuar con el programa, es decir los brotes generados por los árboles tratados no mostraron atributos uniformes. La mayor cantidad de brotes cosechados se obtuvieron de árboles tratados del sitio Shebonia, lo cual confirmaría la mayor capacidad de brotación de los árboles candidatos seleccionados en este sitio.

Ruíz et al., (2005) obtuvieron brotes vigorosos de tamaño uniforme provenientes de tocones de Gmelina arborea manejados en un jardín clonal experimental. El manejo incluyó la aplicación de podas, riegos, control de plagas, control de enfermedades y fertilización. La poda fue total (se eliminaron todos los brotes) y se aplicó 15 días antes de iniciar el experimento, posteriormente se aplicó $100 \mathrm{~g}$ de fertilizante por tocón (60N-00P-44K). Soudre et al., (2010) provocaron la proliferación de brotes fisiológicamente aptos y de tamaño uniforme en tocones de Guazuma crinita de 10 años de edad, mediante el tratamiento del desbaste. Después de 120 días, realizaron la poda total de los brotes (de primera generación), lo que desencadenó la emisión de una gran cantidad de brotes (de segunda generación). Estos brotes fueron manejados bajo sombra (80\%) por 30 días, y finalmente se encontraron fisiológicamente aptos para su cosecha.

Ruíz et al., (2005) y Soudre et al., (2010) obtuvieron brotes vigorosos y de tamaños uniformes provenientes de tocones de especies forestales. Sin embargo, previamente realizaron actividades de manejo y de tratamientos de rejuvenecimiento de los brotes del tocón, lo que permitió la cosecha con las características deseables. Leakey \& Mesén (1991) señalan que en la aplicación del tratamiento del desbaste, las plantas donantes que se cortan a alturas diferentes, desarrollan diferentes números de brotes. A pesar de que el tratamiento de desbaste se ejecutó sobre los $35 \mathrm{~cm}$ del nivel del suelo, se reportó diferencias entre el número de brotes por árbol candidato tratado y las características de los mismos.

Para la obtención de brotes de tamaños uniformes y de características fenotípicas deseables, se deben realizar actividades de manejo y tratamientos de rejuvenecimiento a los árboles candidatos. El manejo debe incluir la adición de fertilizantes y los tratamientos de rejuvenecimiento deben incluir la aplicación de podas. Sin embargo, no es posible aún conocer la dosis del fertilizante específica para cada especie forestal y la cantidad de podas a ejecutarse.

Alfenas et al., (2004) señalan que la capacidad de brotación de los árboles puede variar de acuerdo con el genotipo de la planta, época del año, luminosidad, espesor y profundidad de corte. Siendo las variaciones ambientales mínimas entre los sitios, las respuestas de los árboles candidatos a la aplicación de técnicas de rejuvenecimiento, debió ser afectada en mayor 
proporción por el genotipo de cada árbol. Sin embargo, es necesario mencionar que la población evaluada no fue muy amplia, por lo tanto los resultados obtenidos deben ser considerados como preliminares.

\section{Conclusiones.}

Según la cantidad de brotes observados y cosechados, los árboles candidatos del sitio Shebonia reportan mayor capacidad de brotación, es decir respondieron mejor a la aplicación de los tratamientos de rejuvenecimiento. Se logró una mayor cantidad de brotes (en promedio 5 brotes por árbol tratado), aplicando el tratamiento del desbaste, mientras que con el tratamiento del descortezado se obtuvo un promedio menor a los 2 brotes por árbol. De manera preliminar se puede aseverar que hay un efecto positivo de la aplicación previa de fertilizantes al árbol, sobre la cantidad de brotes observados en los árboles tratados; sin embargo, se recomienda realizar un análisis de suelo más exhaustivo por cada árbol candidato seleccionado, considerar también el efecto de la edad del árbol como factor de variabilidad en la generación de brotes, e incrementar el tamaño de la población para un ensayo posterior, puesto que los criterios de calidad de árbol establecidos para la selección de árboles candidatos, limitaron la posibilidad de incorporar un mayor número de individuos en el tamaño de la población a ser considerada en el diseño de esta investigación.

\section{Agradecimientos.}

Se expresa nuestro más sincero agradecimiento al Consorcio Agroforestal SAC - Grupo PLANTAR, con mención especial al Ing. Fernando Crespo Álvarez, Ing. Cinthya Cáceres Rodríguez, Lic. Evelyn Pinado López, Lic. Guadalupe Poblet Odar, Téc. Miguel Vásquez Vásquez, Eco. Enrique Zárate Villanueva y personal de operaciones. Los profesionales que contribuyen en el Proyecto Innóvate Perú - UNALM (Código del Proyecto: PIPEI-6-P-328-096-13): Ing. Ignacio Piqueras Villarán, Lic. Hilda Flores Zavala, Ing. Diego Castro Garro, Ing. Jorge Luis Aguilar Gálvez e Ing. Catherine Sueros Herrera.

Los profesionales externos que contribuyeron con la investigación: Mg. Sc. Grimaldo Febres Huamán, Dr. Julio Alegre Orihuela, Lic. Patricia Baquerizo Huapaya y Téc. Wilson Saldaña Meléndez.

\section{Literatura citada}

Aguirre C. \& Fassbender D. 2013. Selección de árboles plus de 7 especies forestales nativas de importancia ecológica y económica. Deutsche Gesellschaftfür Internationale Zusammenarbeit (GIZ) y Proyecto de Conservación de Bosques Comunitarios (CBC). Lima, PE, s.e. 60 p.

Alfenas A., Valverde E. Gonçalves R. \& Assis T. 2004. Clonagem e doenças do eucalipto. Viçosa, BR., Universidad Federal de Viçosa. 415 pp.
Assis F. \& Rodríguez F. 2014. Mejoramiento Genético de Eucaliptos en Chile. La propagación vegetativa de eucaliptos en Chile. INFOR - FIA, Chile. p. 125-146.

Bello A. 1997. Procedimiento de selección de árboles plus de roble y raulí. Concepción, $\mathrm{CH}$, s.e. p. 29-36. (Actas $1^{\circ}$ Taller de Mejora Genética en roble y raulí).

DRFFMA (Dirección Regional de Forestal, Fauna y Medio Ambiente). 1991. s.e. Ucayali, PE. s.e.70 p.

Gutiérrez B., Chung P. \& Ipinza R. 1994. Propagación vegetativa y silvicultura clonal en eucalipto. Ciencia e investigación Forestal-Instituto Forestal, Chile 1(8): 140-175.

Gutiérrez B. 1995. Consideraciones sobre la fisiología y el estado de madurez en el enraizamiento de estacas de especies forestales. Ciencia e investigación ForestalInstituto Forestal, Chile 2(9): 261-277.

IIAP (Instituto de Investigaciones de la Amazonia Peruana). 2009. Evaluación económica de parcelas de regeneración natural y plantaciones de bolaina blanca (Guazuma crinita) en el Departamento de Ucayali. Iquitos, PE. 54 p. (Avances económicos $\mathrm{N}^{\circ} 11$ ).

Leakey R. \& Mesén F. 1991. Métodos de Propagación Vegetativa en Árboles Tropicales. Métodos de Propagación en Árboles Tropicales: Enraizamiento de Estacas Suculentas. Turrialba, CR. p 147- 167.

Mesén F., Leakey R. \& Newton A. 1996. Propagadores de sub-irrigación: un sistema simple y económico para la propagación vegetativa de especies forestales. Ed. R Salazar. Managua, NI, s.e. p. 101-110. (Avances en la Producción de Semillas Forestales en América Latina).

Mesén, F. 1998. Enraizamiento de estacas juveniles de especies forestales: uso de propagadores de subirrigación. CATIE. Turrialba, CR. 41 p. (Serie Técnica. Manual Técnico $\mathrm{N}^{\circ} 30$ ).

Nogueria H., Gonçalves A., Trindade C., \& Tamara R. 2011. Cultivo de eucalipto: implantaçao e Manejo. s.e. Viçosa, BR., Aprenda Fácil. 353 p.

ONERN (Oficina Nacional de Evaluación de Recursos Naturales). 1976. Mapa Ecológico del Perú. Guía Explicativa. Lima, PE. 274 p.

Ramos A. 2015. Propagación por estacas de bolaina blanca (Guazuma crinita Mart.) provenientes de árboles candidatos a plus en condiciones de cámara de subirrigación. Tesis Ing. For. Lima, PE, Universidad Nacional Agraria La Molina.130 p.

Reynel C., Pennington RT., Pennington TD., Flores C. \& Daza A. 2003. Árboles útiles de la Amazonía peruana y sus usos: Un manual con apuntes de identificación, ecología y propagación de las especies. Lima, PE, Tarea Gráfica Educativa. 509 p.

Ruiz R., Vargas J., Cetina V. \& Villegas A. 2005. Efecto del ácido indol butírico (AIB) y tipo de estaca en el enraizamiento de Gmelina arborea Roxb. Fitotecnia Mexicana. 4(28): 319-326.

Soudre M., Paredes O., Guerra H., Ríos R. \& Pinedo R. 2010. Tecnología para la producción de estacas juveniles de plantas superiores de bolaina blanca (Guazuma crinita). PROBOSQUES. IIAP. Memoria. s.p.

Torres G. 1987. Almanaque del banco de seguros del estado. El rebrotado en eucaliptus. San Paulo, BR, s.e. 320 p.

Trujillo E. 2009. Guía de Reforestación. 2 edición. Dayber Imprenta. Bogotá, CO. 255 p.

Vallejos J. 2007. Contribuciones al programa de mejoramiento genético de BARCA S.A. en Costa Rica. 
Informe de Graduación de Bachiller Ing. For. Instituto Tecnológico de Costa Rica, Cartago, CR. 80 p. , Badilla Y., Picado F. \& Murillo O. 2010.

Metodología para la selección e incorporación de árboles plus en programas de mejoramiento genético forestal. Agronomía Costarricense 34(1): 105-117.

Villegas P. 2003. Manejo de rebrotes de Eucalyptus globulusLabillardieve en el Altiplano Mexicano. Tesis Ing. For. Universidad Autónoma de Chapingo, Chapingo, MX. 86 p.
Yepes F., Castillo D., Chung J. \& Souza J. 2010. Protocolo para la propagación vegetativa de caoba, Swietenia macrophylla. Cooperación IIAP - FINCyT. s.p. (Memoria).

Xavier A., Wendling I. \& Da Silva R.L. 2013. 2 ed. Silvicultura clonal: Principios e Técnicas. Viçosa, BR., Universidad Federal de Viçosa. 279 p.

Zabaleta A. 1992. Edafología. El suelo en relación con la Producción. Lima, PE., Biblioteca Nacional del Perú. $223 \mathrm{p}$.

${ }^{1}$ Actual: Arborizaciones EIRL, Lima. Anterior: Consorcio Agroforestal SAC, Puerto Súngaro, Puerto Inca, Huánuco.

${ }^{2}$ Profesor principal Facultad de Ciencias Forestales. Universidad Nacional Agraria La Molina. La Molina, Lima. 